\section{ELEKTIVER EINGRIFF NACH MYOKARDINFARKT}

\section{Wie lange warten?}

Eine Auswertung der Daten der California Patient Discharge Database sollte zeigen, wie sich Patienten ohne und mit kürzlich durchgemachtem Herzinfarkt bezüglich postoperativer Komplikationen unterschieden. Dabei ergab sich für Patienten mit einem Myokardinfarkt in den letzten $30 \mathrm{Ta}$ gen: ein erhöhtes Risiko für einen postoperativen Herzinfarkt, eine erhöhte 30-TagesMortalität und eine erhöhte 1-Jahres-Mortalität. Je länger die Chirurgen mit dem elektiven Eingriff warteten, desto geringer wurde das Komplikationsrisiko der Postinfarktpatienten: Bei einem Intervall von bis zu 30 Tagen erlitten 32,6\% der Patienten postoperativ einen erneuten Infarkt. Bei 31-60 Tagen waren es noch $18,7 \%$, bei $61-$ 90 Tagen 8,4\% und bei 91-180 Tagen 5,9\%. Die Studienautoren empfehlen, elektive Operationen nach Möglichkeit frühestens acht Wochen nach einem Myokardinfarkt vorzunehmen.

Ann Surg 2011;253(5):857-864

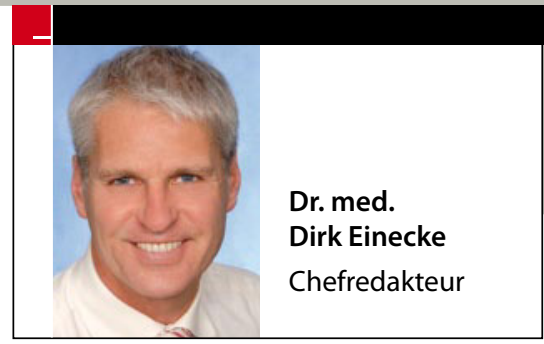

LIPIDSENKER MIT ZUSATZEFFEKT

\title{
Schützen Statine vor dem Prostata-Ca.?
}

Die Vermutung, dass Statine einem Prostatakarzinom vorbeugen können, erhält Unterstützung durch eine neue Studie. In der retrospektiven Untersuchung wurden die Prostatabiopsieraten von Männern mit und ohne Statintherapie verglichen. Ausgewertet wurden die Daten von 4204 Männern, davon 1022 Statinanwender. Alle Männer hatten sich wegen einer positiven digitalrektalen Untersuchung oder wegen erhöhter PSA-Werte einer Prostatabiopsie unterzogen. Ein Prostatakarzinom war bei 55,2\% der Männer unter Statintherapie (mittleres Alter 65,7Jahre) entdeckt worden. Von den Männern ohne Statin (mittleres Alter 63,5 Jahre) hatten 57,8\% ein Prostatakarzinom $(p=0,15)$. Statinanwender hatten seltener einen positiven Befund bei der digital-rektalen Untersuchung (53\% vs. $8,9 \%, p<0,01)$, seltener einen GleasonScore von 7 oder mehr $(61,4 \%$ vs. $72,4 \%$, $p=0,02)$ und seltener ein ausgedehntes Prostatakarzinom mit mindestens drei positiven Stanzen $(27,2 \%)$.

JUrology 2011; 186: 86-90

\section{RISKANTER ZEITVERTREIB}

\section{Wer viel fernsieht, ist früher tot}

Europäer verbringen angeblich 40\%, Amerikaner sogar $50 \%$ ihrer Freizeit vor dem Fernseher. Dafür bezahlen sie mit Diabetes, Herzinfarkt und vorzeitigem Tod. Pro zwei Stunden, die täglich vor dem Fernseher verbracht werden, steigt das Risiko für einen Typ-2-Diabetes um 20\%, das Risiko für eine kardiovaskuläre Erkrankung um 15\% und das Risiko eines vorzeitigen Todes um $13 \%$, zeigt eine Metaanalyse aller prospektiven Kohortenstudien, die zwischen 1970 und 2011 zu diesem Thema veröffentlicht worden sind. Zahlreiche Studie haben gezeigt, dass durch Fernsehen Risikofaktoren für diese Ereignisse wie Adipositas oder Dyslipidämien gefördert würden. Welchen
Anteil Bewegungsmangel und welchen Anteil eine ungesunde Ernährung während des Fernsehens daran haben, sei noch zu klären. Die Forscher fordern außerdem Studien, die den Einfluss der neuen Medien auf das Risiko chronischer Erkrankungen untersuchen.

JAMA 2011;302(23):2448-2455 